\title{
Scientific CCD characterisation at Universidad Complutense LICA Laboratory
}

\author{
Tulloch, $\mathrm{S}^{1^{*}}$., Gil de Paz, $\mathrm{A}^{2}$., Gallego, $\mathrm{J}^{2}$., Zamorano, $\mathrm{J}^{2}$. Tapia, $\mathrm{C}^{2}$. \\ ${ }^{1}$ FRACTAL SLNE, C/ Tulipan 2 Portal 13, 1A, 28231, Las Rozas, Madrid, Spain \\ ${ }^{2}$ Facultad de Ciencias Físicas, Universidad Complutense, 28040, Madrid, Spain
}

\begin{abstract}
A CCD test-bench has been built at the Universidad Complutense's LICA laboratory. It is initially intended for commissioning of the MEGARA ${ }^{1}$ (Multi-Espectrógrafo en GTC de Alta Resolución para Astronomía) instrument but can be considered as a general purpose scientific CCD test-bench. The test-bench uses an incandescent broad-band light source in combination with a monochromator and two filter wheels to provide programmable narrow-band illumination across the visible band. Light from the monochromator can be directed to an integrating sphere for flat-field measurements or sent via a small aperture directly onto the CCD under test for high accuracy diode-mode quantum efficiency measurements. Point spread function measurements can also be performed by interposing additional optics between sphere and the CCD under test. The whole system is under LabView control via a clickable GUI. Automated measurement scans of quantum efficiency can be performed requiring only that the user replace the CCD under test with a calibrated photodiode after each measurement run. A $20 \mathrm{~cm}$ diameter cryostat with a $10 \mathrm{~cm}$ window and Brooks Polycold PCC closed-cycle cooler also form part of the test-bench. This cryostat is large enough to accommodate almost all scientific CCD formats has initially been used to house an E2V CCD230 in order to fully prove the test-bench functionality. This device is read-out using an Astronomical Research Camera controller connected to the UKATC's UCAM data acquisition system.
\end{abstract}

Keywords: scientific CCD, test-bench, quantum efficiency, LICA, MEGARA,UCM

\section{INTRODUCTION}

The test-bench consists of a stable incandescent light source that is wavelength selected using a combination of a colour filter wheel and a computer controlled monochromator. A second filter wheel contains ND filters that allow the intensity of the source to be varied. The wavelength-selected output is then directed along an optical rail onto which various optical elements can be mounted. One possible configuration is to mount an integration sphere on this rail close to the monochromator output and a CCD camera at the downstream end of the rail. The CCD will then experience an even illumination (a "flat-field") that is very useful for detecting cosmetic problems such as bad pixels and column defects. An alternative configuration is to remove the integrating sphere and directly illuminate the CCD to allow measurement of its quantum efficiency $(\mathrm{QE})$. This direct illumination results in a much larger signal: approximately 5 orders of magnitude higher. To measure QE the CCD must be operated in diode mode where it behaves as a single large-area photodiode. The $\mathrm{QE}$ is calculated by ratioing the current that it generates in response to illumination by the monochromator with the current produced by a calibrated photodiode. Since photodiode and CCD will have differing areas it is necessary to pass the monochromator beam through a $5 \mathrm{~mm}$ aperture such that both CCD and photodiode are under-filled by the beam. This $5 \mathrm{~mm}$ aperture effectively defines a collimated beam along the length of the optical rail. If we leave the photodiode in place behind this aperture and then mount optical elements such as filters on the rail we can then use the photodiode signal to measure the spectral transmission characteristics of these filters. It is also possible to mount re-imaging optics on the rail to project test patterns onto a CCD camera. The test pattern can be mounted in the exit aperture of the integrating sphere. This can take the form of an image transparency or a simple pinhole. Images of the assembled system can be found in Figures 1,2 and 3.

*smt@qucam.com; +34 663604482

High Energy, Optical, and Infrared Detectors for Astronomy V, edited by Andrew D. Holland, James W. Beletic, Proc. of SPIE Vol. 8453, 845328 - @ 2012 SPIE · CCC code: 0277-786X/12/\$18 · doi: 10.1117/12.925316 


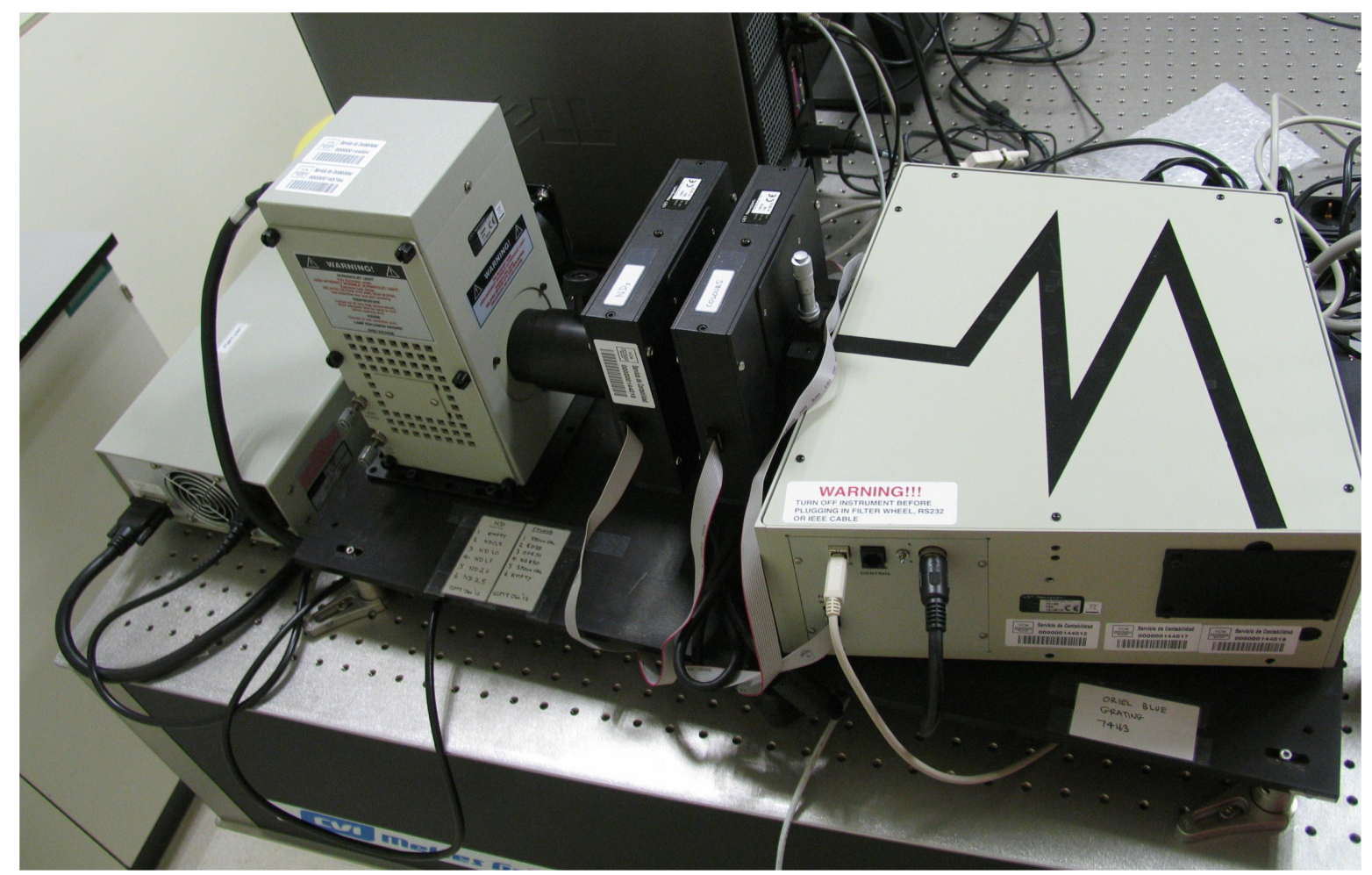

Figure 1. From left to right: stabilized lamp power supply, light source, filters wheels and monochromator.

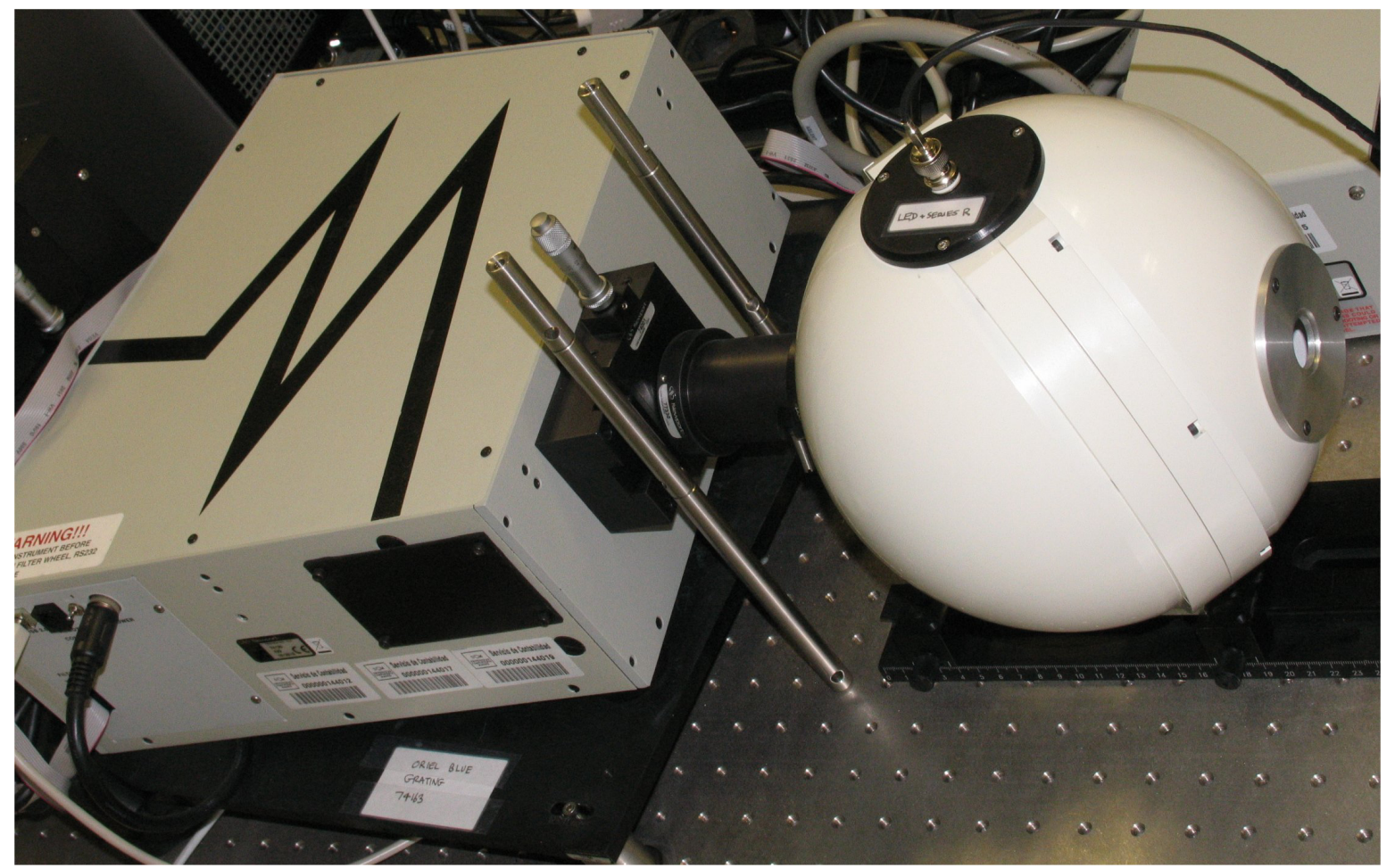

Figure 2. The integrating sphere mounted at the exit of the monochromator. The connector to the internal LED can be seen at the north pole of the sphere. 


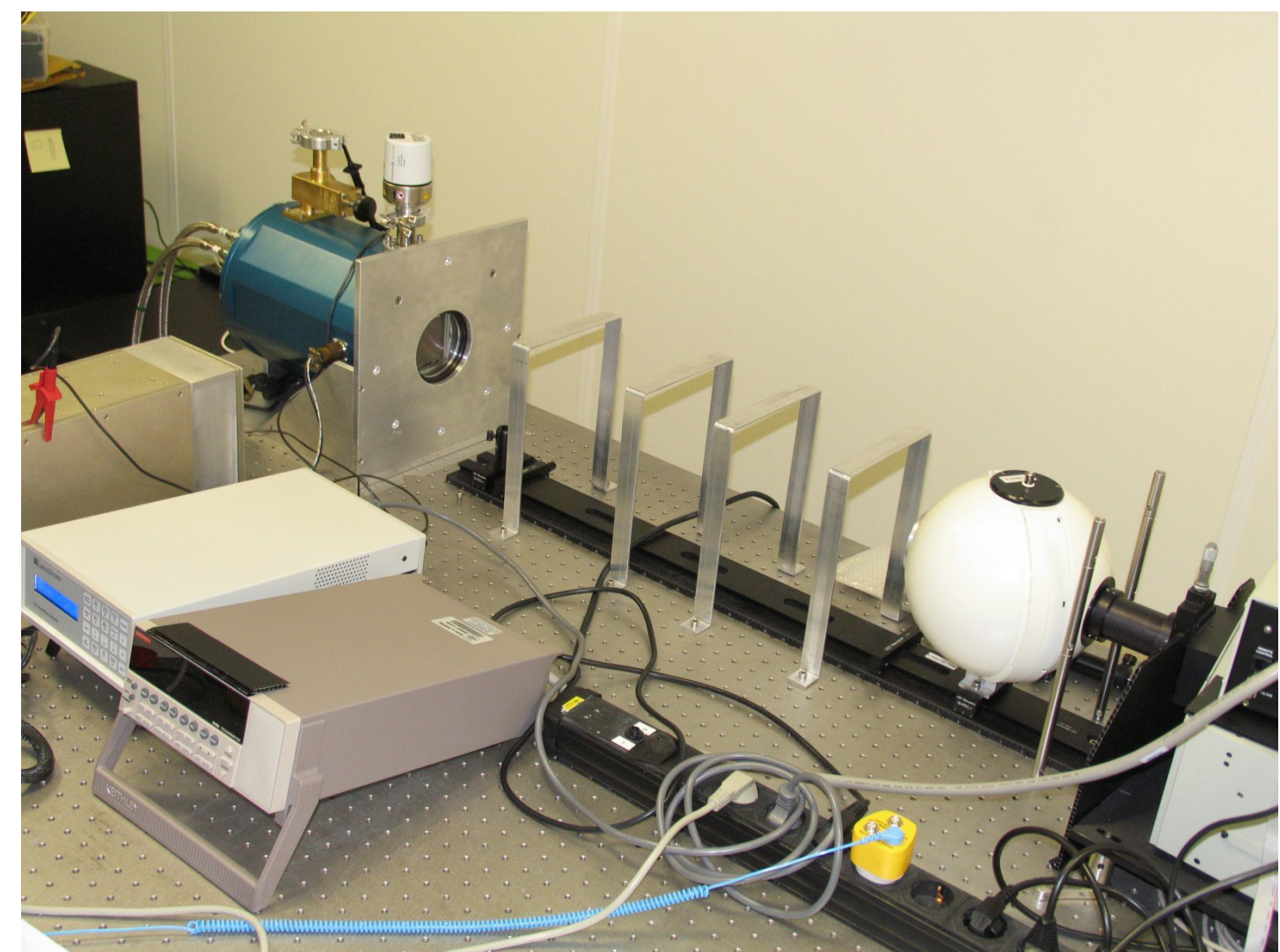

Figure 3. The cryogenic CCD230-based test camera mounted on the test-bench in the flat-field measurement configuration. The metal bridges are to support the opaque cloth that is draped over the test-bench to exclude stray light.

\section{HARDWARE OVERVIEW}

\subsection{Lamp}

A $20 \mathrm{~W}$ Oshio FHE/ESB with a colour temperature of 3200K was selected. A second 50W 3400K lamp from the same Oshio range was also available for applications requiring higher blue-end output. These lamps have lifetimes of less than 100 hours and are expensive, however, cheaper alternatives were found to have no output below $400 \mathrm{~nm}$. The lamp was mounted in a Newport Oriel 66882 housing and passively cooled. It is powered by a stabilized constant-current source.

\subsection{Heat rejection filter}

This filter is of KG2 glass that reaches $50 \%$ transmission at about $800 \mathrm{~nm}$ and $12 \%$ at $1 \mu \mathrm{m}$. It therefore prevents filters further downstream being excessively heated. Unfortunately the loss of flux at $1 \mu \mathrm{m}$ is problematic given that the monochromator's blue-optimised grating already has a low throughput at this wavelength. If large fluxes of NIR light are required this filter can be removed provided that the $50 \mathrm{~W}$ lamp is not in use. Even with the heat rejection filter in place the system is still limited by the blue-end output. This filter becomes too hot to handle when used with the 50W lamp so is clearly doing a good job in protecting more sensitive filters downstream.

\subsection{Neutral density filter wheel}

This is a Newport-Oriel 74041 wheel controlled via a GPIB interface. It has 6 positions 5 of which are loaded with filters ranging from ND 0.5 to ND 2.5. It was intended as a way of varying the source intensity although in practice it is always used in the open position so as to maximize flux. It is mounted downstream of the heat-absorption filter. 


\subsection{Color filter wheel}

This is a Newport-Oriel 74010 wheel controlled via a direct cable connection from the monochromator. It has 6 positions 5 of which are loaded with colour filters. It is mounted immediately upstream of the monochromator. Filters currently present are: $390 \mathrm{~nm}$ line filter, 550nm line filter, BG38 blue pass, OG570 red pass and RG830 IR pass. The first two of these are intended for wavelength calibration and scattered light checks, the final three are to reduce scattered light and second order leakage from the monochromator grating. The BG38 filter is essential for reducing scattered light when the monochromator is used below $570 \mathrm{~nm}$. In this region the lamp output is very low compared to its peak emission at the red end and scattered light would otherwise contribute a significant fraction of the flux at the monochromator output.

\subsection{Monochromator}

This is a Newport-Oriel Cornerstone 260 unit controlled over a USB interface. It is loaded with a 1200 lines per mm grating optimized for blue throughput (Newport-Oriel 74163). The input and output slits were set to a width of $1.6 \mathrm{~mm}$ which corresponds to a bandwidth of $5 \mathrm{~nm}$. For measurements requiring higher spectral resolution this slit width can be manually reduced at the expense of signal using the micrometers. The dispersion of the grating varies slightly across the spectrum. At $350 \mathrm{~nm}$ it is $3.2 \mathrm{~nm}$ per $\mathrm{mm}$, at $1000 \mathrm{~nm}$ it is $2.91 \mathrm{~nm}$ per $\mathrm{mm}$. The monochromator centre wavelength was under LabView control. Scans through wavelength were easy to implement in software. The wavelength accuracy was quoted as $1 \mathrm{~nm}$ in the data sheet. This was later confirmed by our measurements of an emission-line source.

\subsection{Integrating sphere}

This is a Newport-Oriel 70677 8" sphere with a "straight-through" geometry. It contains an internal baffle to prevent the direct transit of un-diffused light. The output port of the sphere has by default a $20 \mathrm{~mm}$ diameter aperture. A $4 \mathrm{k} \mathrm{x} 4 \mathrm{k}$ $15 \mu \mathrm{m}$ pixel detector placed $500 \mathrm{~mm}$ downstream experienced illumination non-uniformity of $<0.5 \%$. The exit aperture can be removed and replaced with either a $1 \mathrm{~mm}$ aperture or a $25 \mu \mathrm{m}$ pinhole. Additionally the sphere has an internallymounted $465 \mathrm{~nm}$ LED that can be pulsed to give high-intensity short-duration flat field illumination. This is useful for linearity measurements of the $\mathrm{CCD}$ and the signal processing chain since the light delivered is closely proportional to the LED "on-time". The sphere is mounted directly at the output of the monochromator.

\subsection{Reference photodiode and picoammeter}

This is a $10 \mathrm{~mm}$ diameter Hamamatsu S2281. Its package has an integral BNC connector permitting direct connection to the Keithley 6485 picoammeter via a low-noise coaxial cable. The photodiode was calibrated by the National Physical Laboratory. The calibration took the form of QE measurements at $20 \mathrm{~nm}$ intervals between $350 \mathrm{~nm}$ and $990 \mathrm{~nm}$ with an extra data point at $1000 \mathrm{~nm}$. Above $390 \mathrm{~nm}$ the calibration data was a smoothly varying function so additional data points were later interpolated. The Keithley is controlled via a GPIB interface. It was configured to make multiple readings of the photodiode current in order to drive down the read noise and to allow estimates of signal to noise ratio (SNR). Each single current measurement took $40 \mathrm{~ms}$ and had a read-noise of $0.2 \mathrm{pA}$. Normally, 50 such readings were averaged to maximize SNR. The main purpose of the photodiode is as a reference when measuring the quantum efficiency of a detector. This process is explained in more detail in the next section.

\subsection{Quantum efficiency measurement method}

Quantum Efficiency is measured by directly projecting an image of the monochromator exit-slit onto a $5 \mathrm{~mm}$ aperture at the far end of the optical rail. Light passing through this aperture is intercepted and measured by the CCD. The CCD is then replaced with a photodiode to calibrate the flux. The CCD quantum efficiency is then calculated by ratioing the signal seen by the CCD with that seen by the photodiode. The assumption is made that the lamp has not changed brightness in the meantime; something that can be confirmed by making photodiode measurements both before and after the CCD measurements. The most accurate way to measure the CCD signal is to treat the CCD as if it were a single large photodiode. This can be done by disconnecting any readout electronics and connecting a picoammeter between the Dump Drain and Substrate pins. For some CCDs it is necessary instead to connect between Reset Drain and Substrate.

Figure 4 shows a CCD camera being measured. The camera is visible in the background with its entrance window partially obscured by the metal plate that holds the $5 \mathrm{~mm}$ aperture. The monochromator slit has been imaged onto this 
aperture using a $50 \mathrm{~mm}$ diameter $150 \mathrm{~mm}$ f.1. lens (also visible in the photo) mounted on the optical rail between camera and monochromator. Notice that the green patch of light that is the monochromator slit image overfills this aperture. Whatever light passes through this aperture then produces a photocurrent in the CCD. Figure 5 shows the region of the aperture in more detail, this time with the calibration photodiode mounted behind the aperture and intercepting all the light that passes through it. The measurement process involves first scanning through the wavelength range of the monochromator and measuring the photodiode current at each wavelength step. The photodiode is then dismounted from the aperture and disconnected from the picoammeter. The CCD is then connected and the wavelength scan repeated. At each wavelength the CCD QE is calculated by dividing its current by the photodiode current and then multiplying by the photodiode QE.

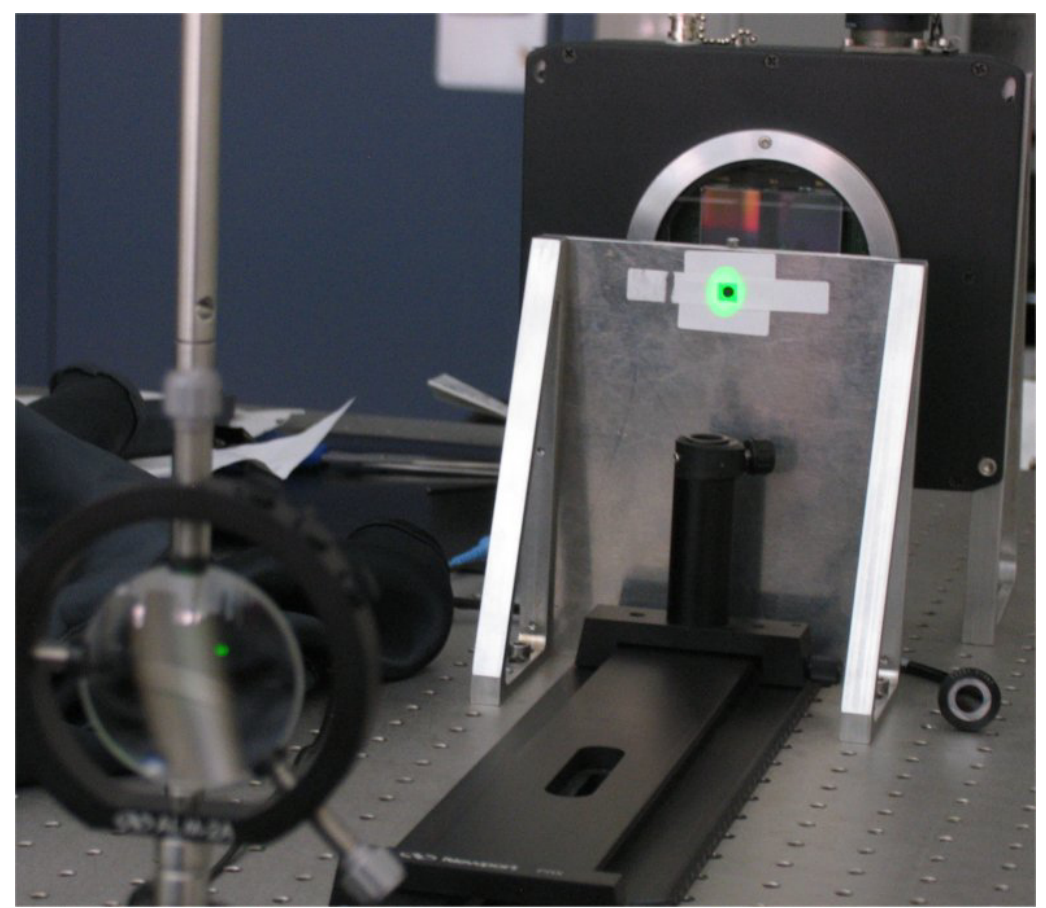

Figure 4. A CCD camera having its QE measured. The monochromator output can be seen focused onto a $5 \mathrm{~mm}$ aperture placed just in front of the camera. The unused photodiode is visible on the optical bench at the right.

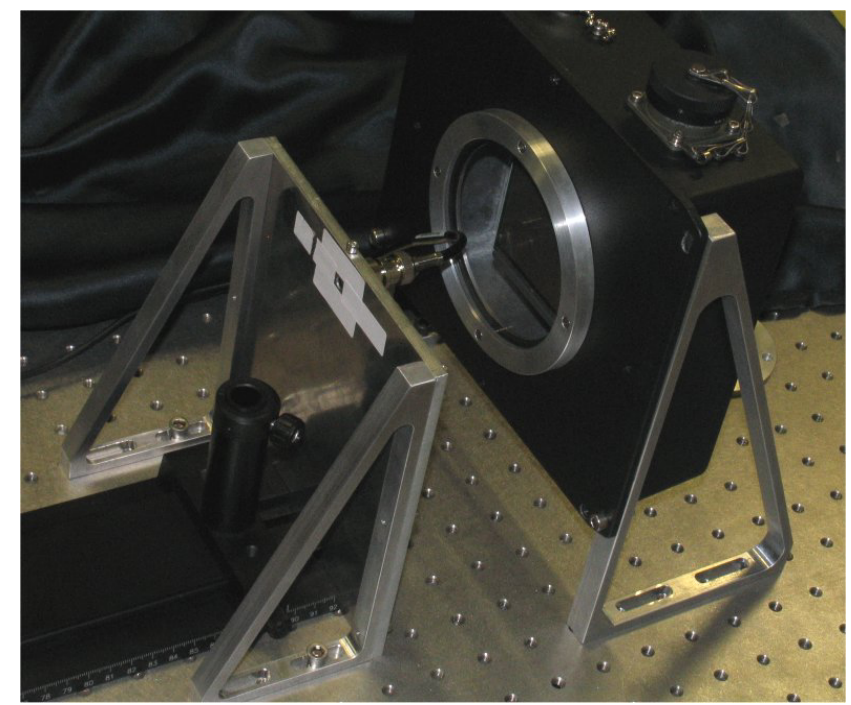

Figure 5. A close up of the $5 \mathrm{~mm}$ aperture support. Here the photodiode has been mounted directly behind the aperture so as to calibrate the light that passes through it. 


\subsection{The cryogenic test camera}

An important part of the test-bench is an IR Laboratories cryostat containing a Polycold (Cryotiger) Joule Kelvin closed cycle cooler. This cooler went down to $93 \mathrm{~K}$ with no load and could pump $22 \mathrm{~W}$ at $173 \mathrm{~K}$. The cryostat has a $10 \mathrm{~cm}$ diameter window and a space of $15 \mathrm{~cm}$ between the inner surface of the window and the cold finger of the cooler. The internal diameter of the cryostat is $20 \mathrm{~cm}$. It is therefore roomy enough for most scientific CCDs. An engineering grade E2V Technologies CCD230 was purchased and mounted in this cryostat. This CCD has a ceramic package that necessitates the use of a head-PCB containing sockets for the CCD pins and a Glenair 37-way micro-D connector. It also contains some Zener diodes to protect the CCD from static discharge. Figure 3 showed the camera mounted on the test-bench. Figures 6,7 and 8 show some additional details.

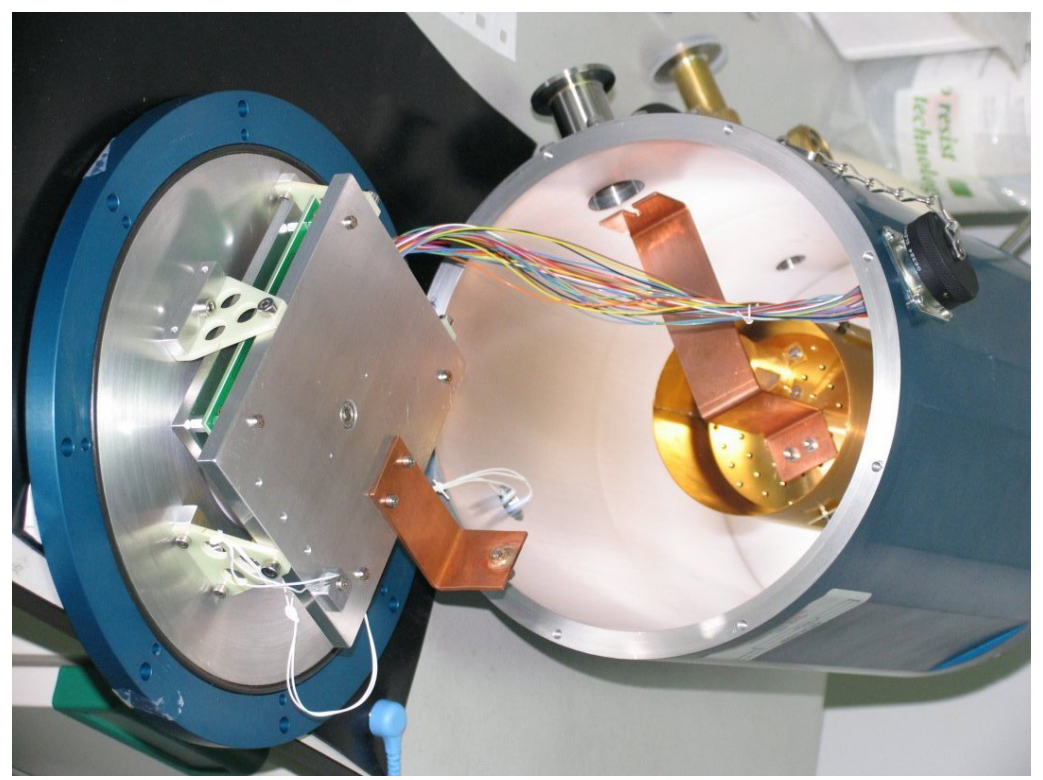

Figure 6. A close up of the CCD230 camera internals. The CCD baseplate is visible on the left connected to the inner surface of the cryostat lid via G10 pillars. On the right can be seen the cold finger. Two copper strips, joined with a bolt during cryostat assembly, complete the thermal link between cooler and CCD.

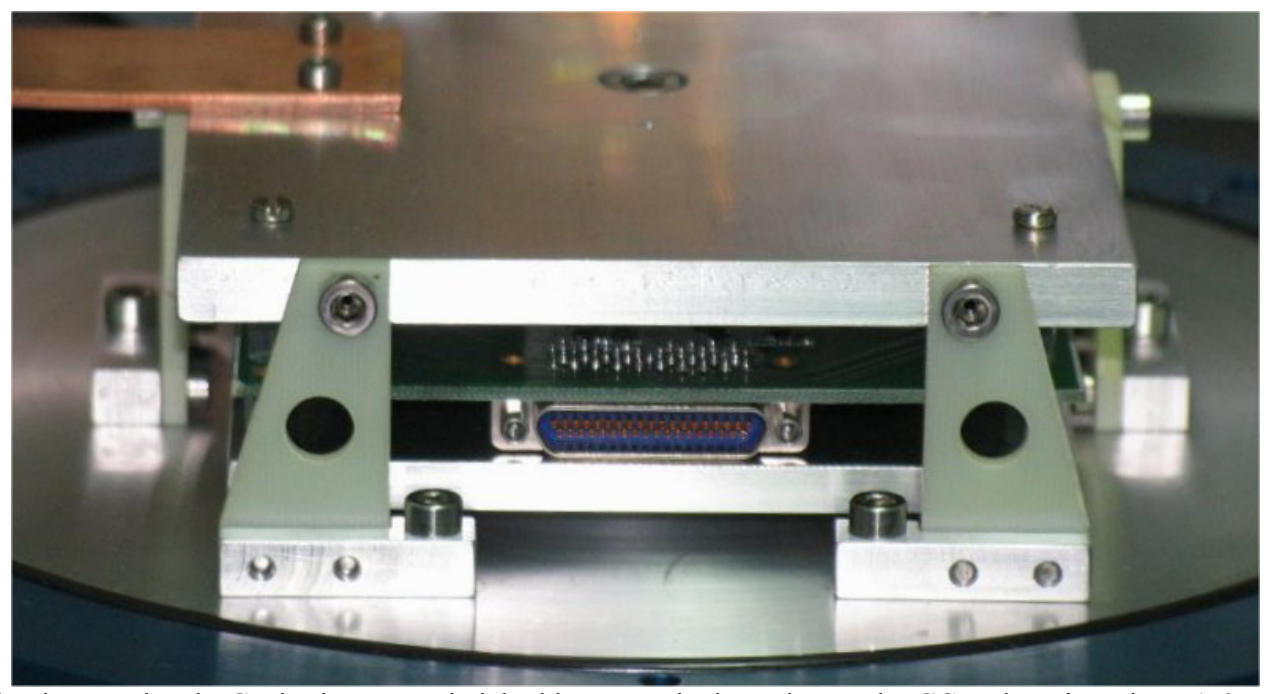

Figure 7. The detector head PCB is shown sandwiched between the baseplate and a CCD clamping plate. A 37-way Glenair connector carrying all CCD signals is also visible at the centre. 


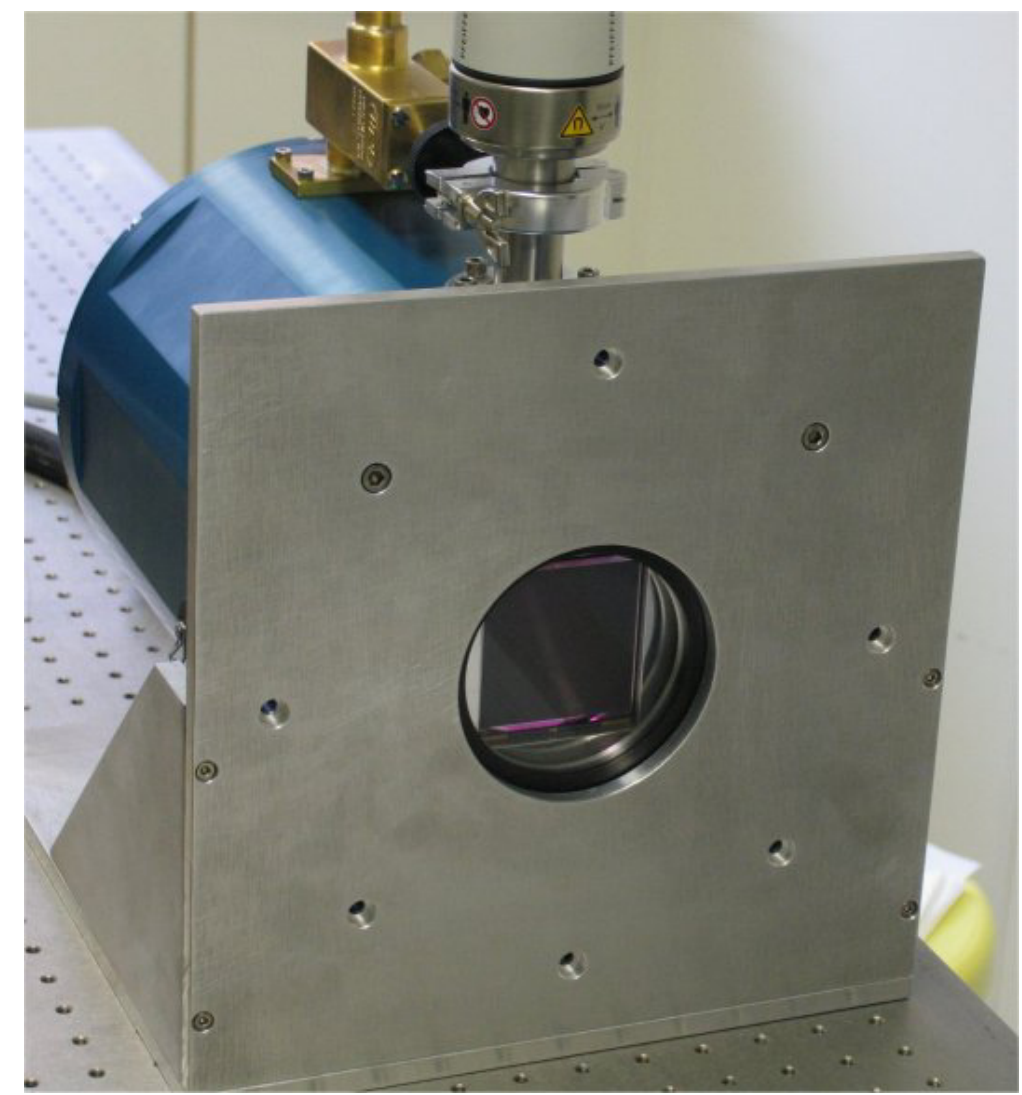

Figure 8. The completed CCD230 camera is shown on its support structure. A vacuum gauge and the vacuum valve are visible along the top of the cryostat.

The test camera is operated with an ARC controller. A four channel video card is used. The ARC was interfaced to an Amplion Impact-R Linux machine running the UCAM data acquisition software provided by the UKATC. Temperature servoing is performed with a Lakeshore 325 controller.

\section{CONTROL SOFTWARE}

\subsection{Software overview}

The test-bench is controlled over the USB and GPIB interfaces using a Windows PC running LabView software. The Newport Oriel Monochromator and filter wheels came with their own libraries of LabView software but in the case of the filter wheel this was found to be out of date and unusable. Custom LabView routines were written to allow low-level commands to be sent to filter wheels and monochromator and these were later incorporated into a control GUI. Three GUI programs were written. The first was for manual positioning of the grating and the filter wheels as well as spot measurements with the Keithley picoammeter. A second GUI allows continuous monitoring of photodiode current which proved useful when setting up for QE measurements and hunting for light leaks. A third LabView GUI performs automated scans and was used for measuring $\mathrm{CCD}$ QE and filter transmissions. This is described in the next section.

\subsection{Control GUIs}

Figure 9 shows a screen capture of the LabView GUI used to perform QE scans. The program scans the full range of 350 to $1050 \mathrm{~nm}$. The user only has to select the wavelength step size. As the scan progresses the program continuously displays the measurement value and its associated RMS noise. The colour filters are automatically changed as the scan 
progresses so as to minimise scattered light (see section 2.4). Once the scan is complete a graph of current versus wavelength is displayed. This provides a sanity check before progressing further with data analysis.

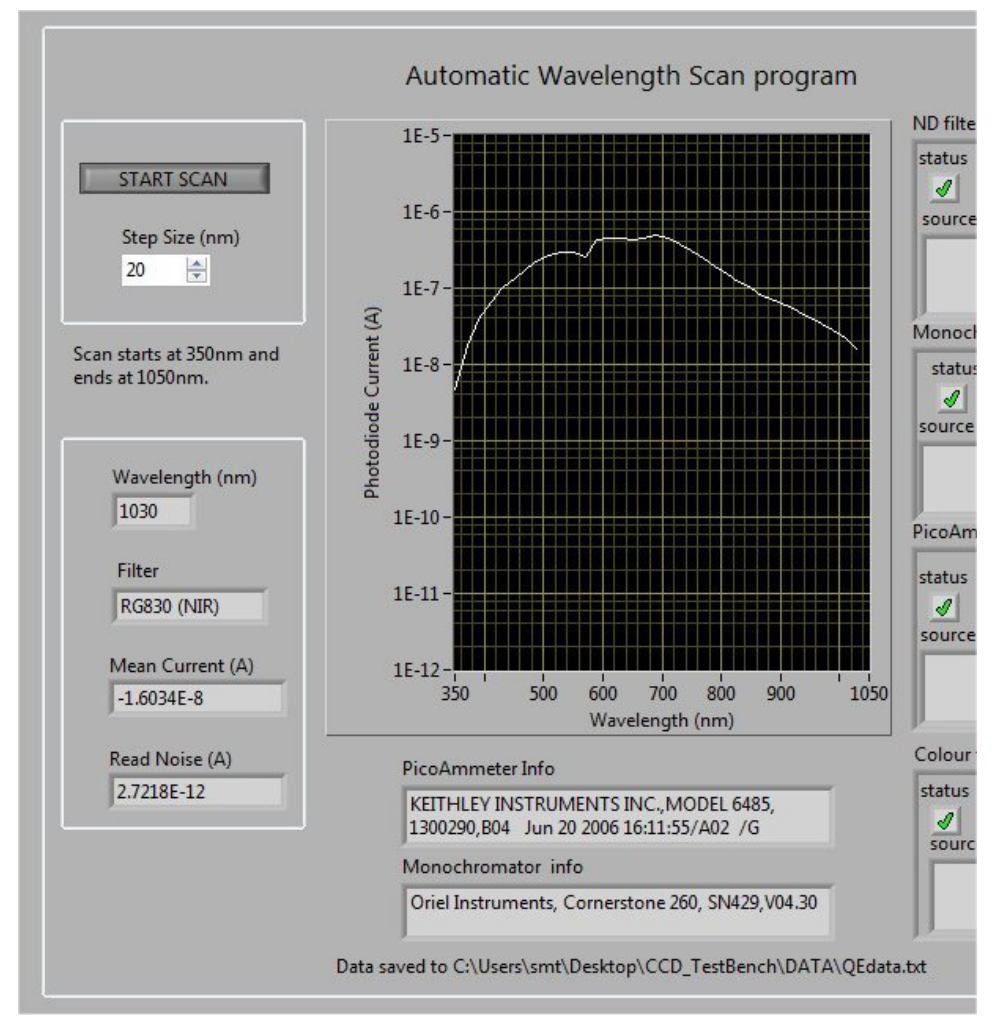

Figure 9. One of the LabView GUIs used to control the test-bench. This GUI was used for automated wavelength scans.

\section{TEST-BENCH CHARACTERISATION}

The flux levels and stability of the test-bench were thoroughly investigated. The wavelength calibration for the monochromator was also checked. The relatively low blue output of the lamp and monochromator compared to the high red-end output of the incandescent lamp made scattered light a key issue in the test-bench design. Measurements were made using additional colour glass filters to prove that scattered light was not significant.

\subsection{Wavelength accuracy}

The lamp housing was opened and the lamp removed. In its place was put a diffusing screen that was then illuminated by a Neon vapor lamp. A wavelength scan was then made with the photodiode at the exit slit of the monochromator. The measured positions of the emission lines were compared with their book values. The Neon spectrum obtained is shown in Figure 10. Neon emits principally in the red. In order to have some data elsewhere in the spectrum a further scan was done with the diffusing screen illuminated by a $532 \mathrm{~nm}$ solid-state laser. Wavelength errors varied between $-0.5 \mathrm{~nm}$ and $-1.2 \mathrm{~nm}$ for the various lines measured. 


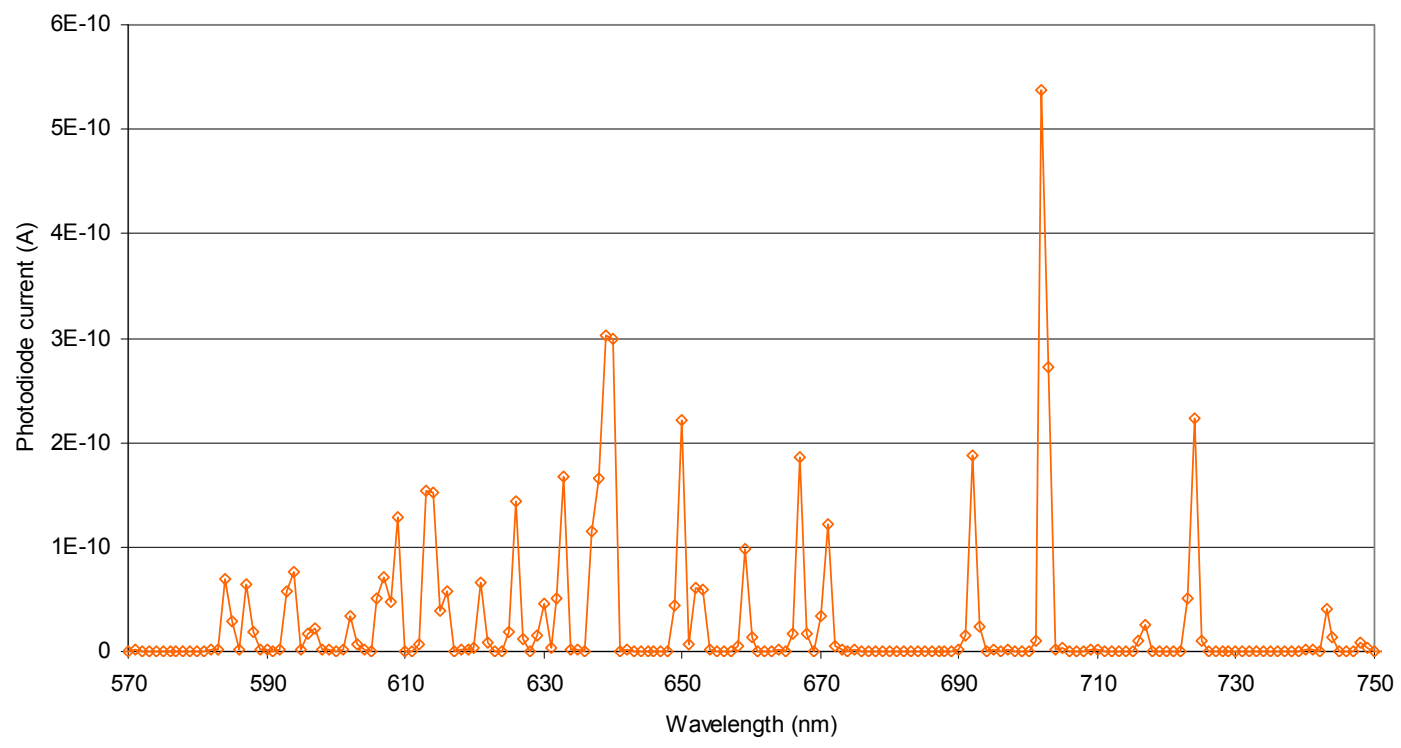

Figure 10. A calibration scan of a Neon emission lamp.

\subsection{Scattered light}

Scattered light in the monochromator system will degrade the spectral purity of the output signal. This will be a particular problem in the blue end of the spectrum where even a small amount of scattered red or green light could overwhelm the low blue output of the system. One way to quantify scattered light is to measure the signal present out-ofband when scanning the transmission of a narrow band filter. This would require a filter with an exceptionally low leakage compared to that of the monochromator. None could be found in the Newport catalogue with a leakage smaller than $10^{-4}$ so an alternative method was devised. To get an idea of the level of scattered light a UG1 filter was taped over the photodiode aperture and it was illuminated with a $532 \mathrm{~nm}$ laser-diode. The photodiode current was then recorded. The UG1 filter was then removed and the photodiode current measured once again. The laser had to be attenuated with an ND 3.5 filter so as not to saturate the photodiode (which becomes non-linear above a few $\mathrm{mA}$ ). These two measurements allowed the transmission of the UG1 at $532 \mathrm{~nm}$ to be precisely measured since the source was monochromatic at $532 \mathrm{~nm}$ and the geometry of the illumination (with the filter taped over the front of the photodiode) eliminated all scattered light. The measured transmission was $1.7 \times 10^{-5}$. Next, the laser-diode was removed and the UG1 filter mounted in a conventional mount on the optical rail a short distance in front of the photodiode aperture (a more standard arrangement). The UG1 filter was then scanned using the monochromator in combination with its colour filter wheel as normal, and its transmission calculated. The transmission at $532 \mathrm{~nm}$ was then compared with the "correct" value measured earlier using the laser. As can be seen from Figure 11 the measured transmission agreed well with the laser data. This shows that the scattered light in the system, comprising light from the lamp that has somehow bypassed the grating and not been blocked by the colour filter wheel, is negligible. UG1 was particularly useful in this test since it allowed the peak wavelength of the lamp output (where we would expect most scattered light to be) to pass with fairly high transmission.

\subsection{Flux output}

When configured to measure $\mathrm{QE}$, the signal from the photodiode peaks at $1 \mu \mathrm{A}$, falling to $10 \mathrm{nA}$ at $350 \mathrm{~nm}$. This measurement was obtained using a $20 \mathrm{~W}$ lamp and a $1.6 \mathrm{~mm}$ wide $(5 \mathrm{~nm}$ band-pass) entrance and exit slit. This is more than bright enough to give high SNRs given that a single photodiode measurement has a noise of $0.2 \mathrm{pA}$. Figure 12 shows the photodiode signal across the whole spectrum. When measuring flat-fields with the integrating sphere, fluxes drop by a large factor. For example with the same slit width and lamp power, a $15 \mu \mathrm{m}$ CCD pixel placed $665 \mathrm{~mm}$ from the $20 \mathrm{~mm}$ diameter exit aperture of the sphere received 940 photons per second at $700 \mathrm{~nm}$ and only 40 photons per second at $390 \mathrm{~nm}$. For blue-end flat-fields it is therefore necessary to use the $50 \mathrm{~W}$ lamp and/or increase the slit width. 


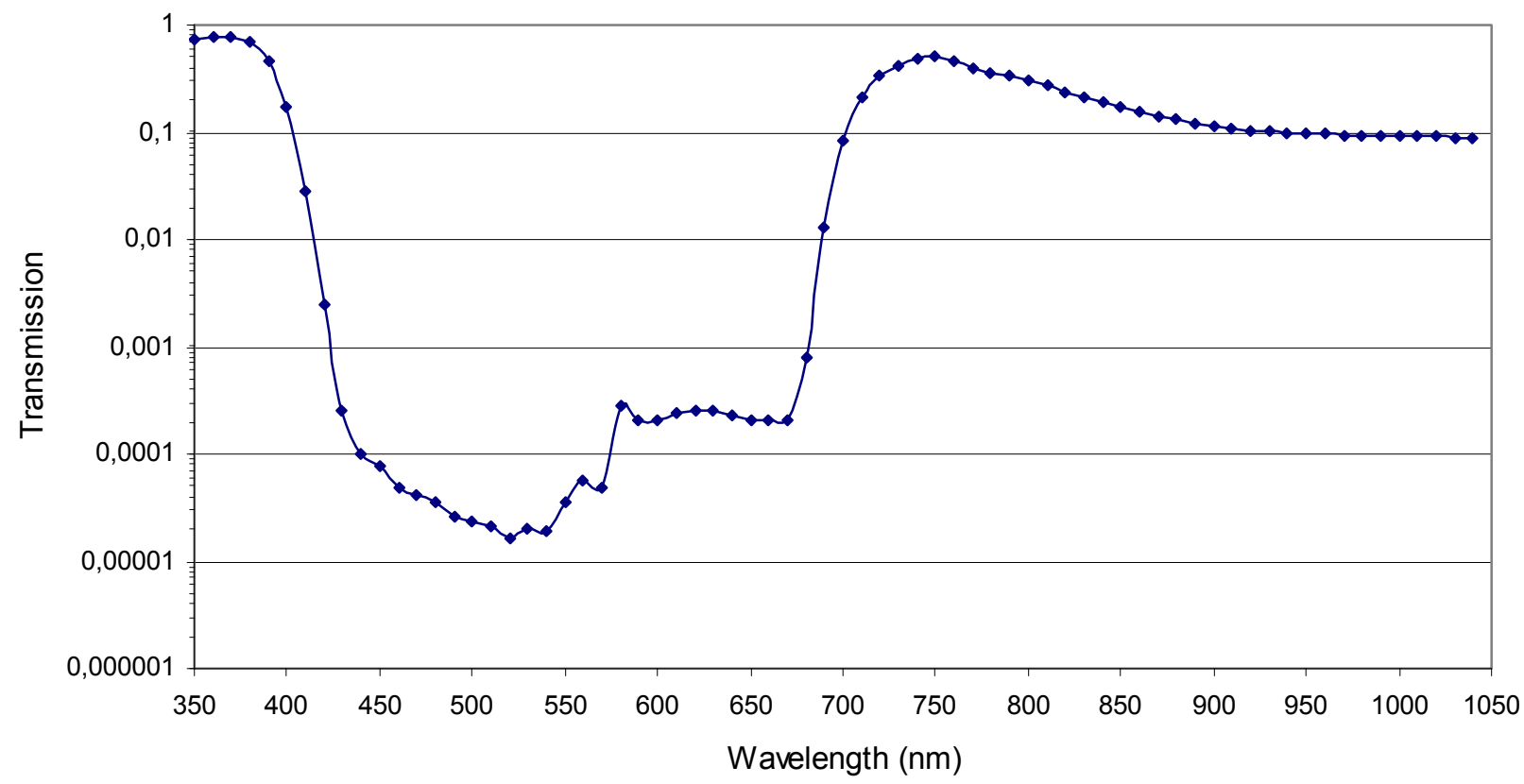

Figure 11. A transmission scan of a UG1 colour glass filter using the test-bench. This filter had a known transmission of $1.7 \times 10^{-5}$ at $532 \mathrm{~nm}$; the same value as obtained by the test-bench.

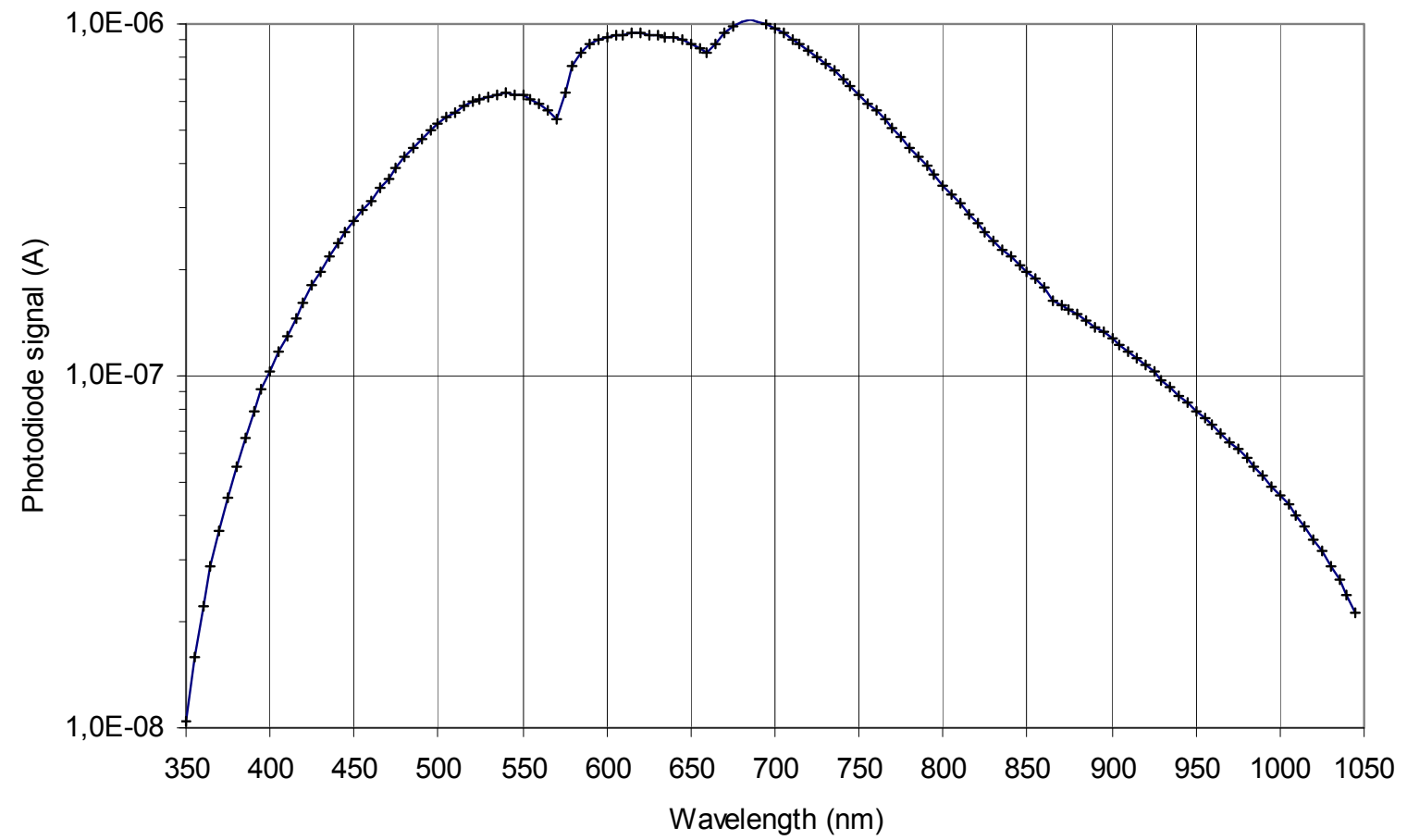

Figure 12. The output of the monochromator. The flux was measured using the photodiode placed behind the $5 \mathrm{~mm}$ aperture used for the QE and filter transmission measurements. The $20 \mathrm{~W}$ lamp and $1.6 \mathrm{~mm}$ wide input and output slits were used.

Proc. of SPIE Vol. $8453845328-10$ 


\subsection{Measurement noise}

A single photodiode measurement had a noise of 0.2pA RMS if the Keithley was configured to integrate on the signal for $40 \mathrm{~ms}$. Background illumination in the laboratory from light leaks under the lab doors and shutters as well as from the various LEDs generally present in laboratory and computing equipment pushed the photodiode signal up to several pA even when the optical bench was covered with an opaque black cloth. The residual photodiode dark current fell to $0.2 \mathrm{pA}$ once these various stray light sources had been taped over. When measuring the CCD photocurrent the Keithley gave a much higher read noise, probably due to the CCDs higher capacitance. For the CCD230 the lowest read noise obtained was $0.12 \mathrm{nA}$ RMS using an acquisition time of $50 \mathrm{~ms}$.

\subsection{System stability}

The lamp took some time to warm up. Its brightness across the whole spectrum was measured 10, 30, 60 and 90 minutes after power-on. Even after 30 minutes the blue end output had not fully stabilized. Between 60 and 90 minutes the lamp brightness had not changed by more than $0.25 \%$ at any wavelength. A 1-hour warm up was therefore recommended prior to doing any measurements. The Keithley picoammeter manual also recommended a 1-hour warm up.

\section{RESULTS FROM TEST-BENCH}

\subsection{Quantum efficiency of a warm CCD230}

The engineering grade CCD230 was measured at room temperature. No allowance was made for losses in the uncoated fused silica window that protected the CCD. The data is shown in Figure 13.

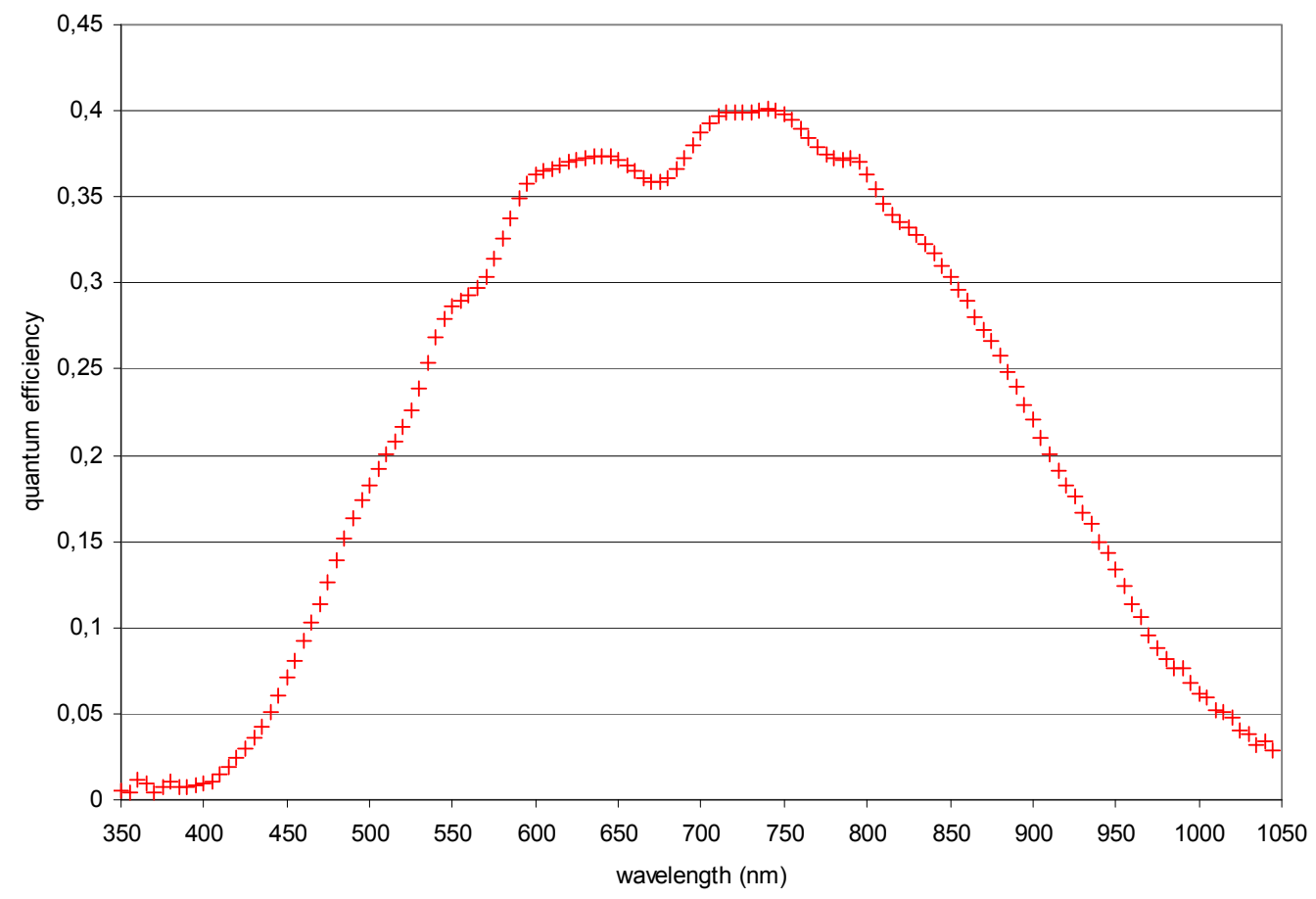

Figure 13. The measured quantum efficiency of a room-temperature CCD230. No allowance has been made for losses in the uncoated camera window. 


\subsection{Measuring filter transmission}

The test-bench lends itself very easily to the measurement of filter transmissions. The photodiode is left mounted behind the $5 \mathrm{~mm}$ aperture and two wavelength scans are done, first with the diode directly illuminated by the monochromator and then with the aperture covered by the filter under test. Figure 14 shows data obtained for a Bayer colour separation filter set.

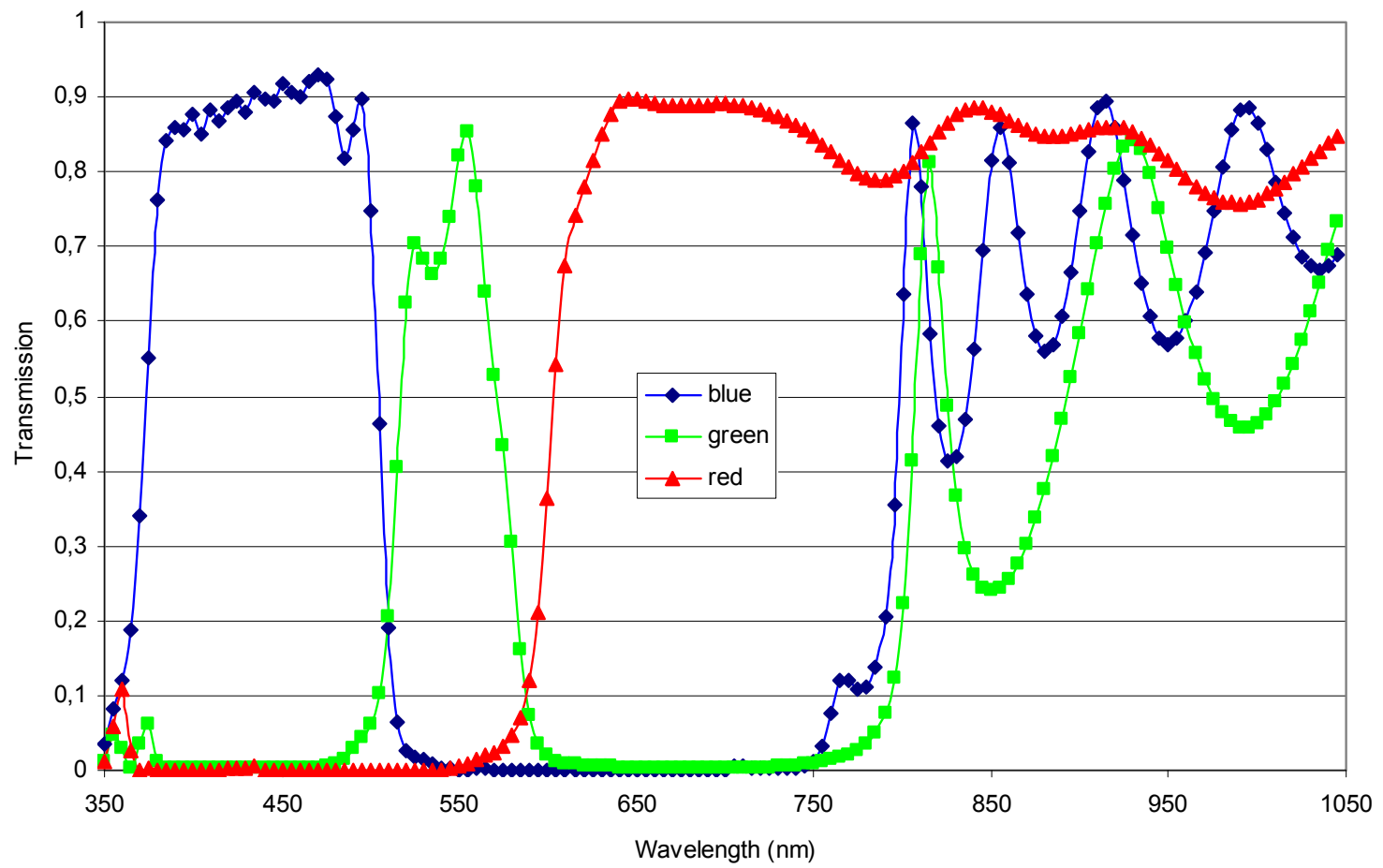

Figure 14. Measured filter transmissions for a Bayer colour separation filter set.

\section{CONCLUSIONS}

The test-bench has been thoroughly characterised and is working as intended. The wavelength calibration is accurate to $1 \mathrm{~nm}$ and levels of scattered light are low enough to enable filter transmissions as low as $2 \times 10^{-5}$ to be measured. The test-bench has been used to obtain good-quality QE and filter transmission data. The integrating sphere gives good flatfields and its internally mounted LED has proved very useful for rapid measurements of linearity. It remains to implement additional optics that will allow the projection of artificial stars onto the CCD under test. Once complete the system will be capable of characterising all of the most significant performance parameters of scientific CCD cameras.

This work was supported by the Spanish Programa Nacional de Astronomía y Astrofísica (project AYA2009-10368) and by the Spanish MICINN under the Consolider-Ingenio 2010 Program grant CSD2006-00070: "First Science with the GTC" (http://www.iac.es/consolider-ingenio-gtc/).

\section{REFERENCES}

[1] Gil de Paz, A, et Al., "MEGARA: the future optical IFU and multi-object spectrograph for the $10.4 \mathrm{~m}$ GTC telescope", Proc. SPIE 8446-182, (2012). 